\title{
PROTECTIVE EFFECT OF NATURALLY-DERIVED ANTIOXIDANTS AGAINST ACETAMINOPHEN-INDUCED HEPATOTOXICITY: A REVIEW
}

\author{
Md. Mizanur RAHAMAN ${ }^{1}$, Shabnam SHARMIN ${ }^{1}$, Olubunmi ATOLANI ${ }^{2}$, Oluyomi Stephen
} ADEYEMI $^{3}$, Mohammad Torequl ISLAM ${ }^{* 1}$

${ }^{1}$ Department of Pharmacy, Bangabandhu Sheikh Mujibur Rahman Science and Technology University, Gopalganj (Dhaka)-8100, Bangladesh

${ }^{2}$ Department of Chemistry, University of Ilorin, P.M.B. 1515, Ilorin, Nigeria

${ }^{3}$ Department of Biochemistry, Medicinal Biochemistry, Infectious Diseases, Nanomedicine \& Toxicology Laboratory, Landmark University, PMB 1001, Omu-Aran 251101, Kwara State, Nigeria

*Correspondence:

Mohammad Torequl ISLAM

dmt.islam@bsmrstu.edu.bd

Received: 20 November 2020; Accepted: 18 December 2020; Published: 30 December 2020

\begin{abstract}
Acetaminophen (APAP) is a commonly used over-the-counter (OTC) drug known to induce hepatotoxicity when consumed in excess. Formation of reactive oxygen species (ROS) and oxidation of cellular proteins and enzymes are directly involved in its toxic mechanisms. However, antioxidants can be helpful to inhibit or restrict the oxidative damage. Besides synthetic antioxidants, naturally-derived substances can be used to serve the purpose. In this paper, a thorough literature review revealed that APAP combined with food-derived natural antioxidants exhibit a protective effect against APAP-induced hepatotoxicity.
\end{abstract}

Keywords: acetaminophen, chemoprevention, hepatotoxicity, free radical, natural antioxidants, redox homeostasis.

\section{Introduction}

Acetaminophen (APAP) is a well-known and frequently used antipyretic and analgesic drug by people all over the world (Bessems et al., 2001; Dargan \& Jones, 2002; James et al., 2003; Yang et al., 2013). It is normally prescribed to relieve conditions such as mild to moderate pain from headaches, muscle aches, toothaches, backaches, menstrual cramp, common colds, sore throats, reaction to vaccines and fever reduction (Medline Plus drug information). At therapeutic dose, acetaminophen is conjugated to glucuronic acid and eliminated in bile as part of metabolism.
Nevertheless, $7.0 \mathrm{mg} /$ day for adults and 150 $\mathrm{mg} / \mathrm{kg}$ for children are considered to be toxic for the liver (Hazai et al., 2002; Kon et al., 2007).

The liver is one of the most vital organs of the body, carrying out over 500 functions including metabolism of ingested substances and detoxification of toxic substances (Almeer et al., 2018). Extensive use of acetaminophen in thousands of prescriptions and over-thecounter drugs has escalated the risk of hepatotoxicity (Larson, 2007; Hinson et al., 2010). 
Though the exact mechanism of APAPinduced liver injury is yet unclear, the idea is that hepatotoxicity starts from the moment its metabolic activation is set in motion. About 95\% of the therapeutic dose is converted into inactive metabolites however, CYP1A2, CYP2E1 coverts the rest of the dose into a toxic metabolite named $\mathrm{N}$-acetyl- $p$-benzoquinone imine (NAPQI) (Corcoran et al., 1985; Esterline et al., 1989; Nelson, 1990; James et al., 2003) (Fig. 1.). In some cases involving APAP overdose, alcohol abuse, hepatic impairment, and starvation, a low reduced glutathione (GSH) level is seen which exponentially multiplies the pernicious effect of NAPQI. As the GSH level is always low, the
NAPQI targets cytosolic and mitochondrial proteins and lipids and disrupts the function of several pro (Bcl-Xs, Bad, Bax, Bid, BAK, BIM) and anti-cell death (Bcl-2, Bcl-XL) genes. This series of incidences gradually leads to mitochondrial dysfunction and results in hepatotoxicity and hepatocellular death (Ray \& Corcoran, 2009; Ghosh et al., 2010; Jaeschke et al., 2012; McGill et al., 2012). NAPQI on the other hand can also increase the formation of reactive oxygen species (ROS) that includessuperoxide ions, hydrogen peroxide, and hydroxyl radical leading to lipid peroxidation and decreased antioxidant enzymes (Michael et al., 1999; Hinson et al., 2002; Hinson 2004).

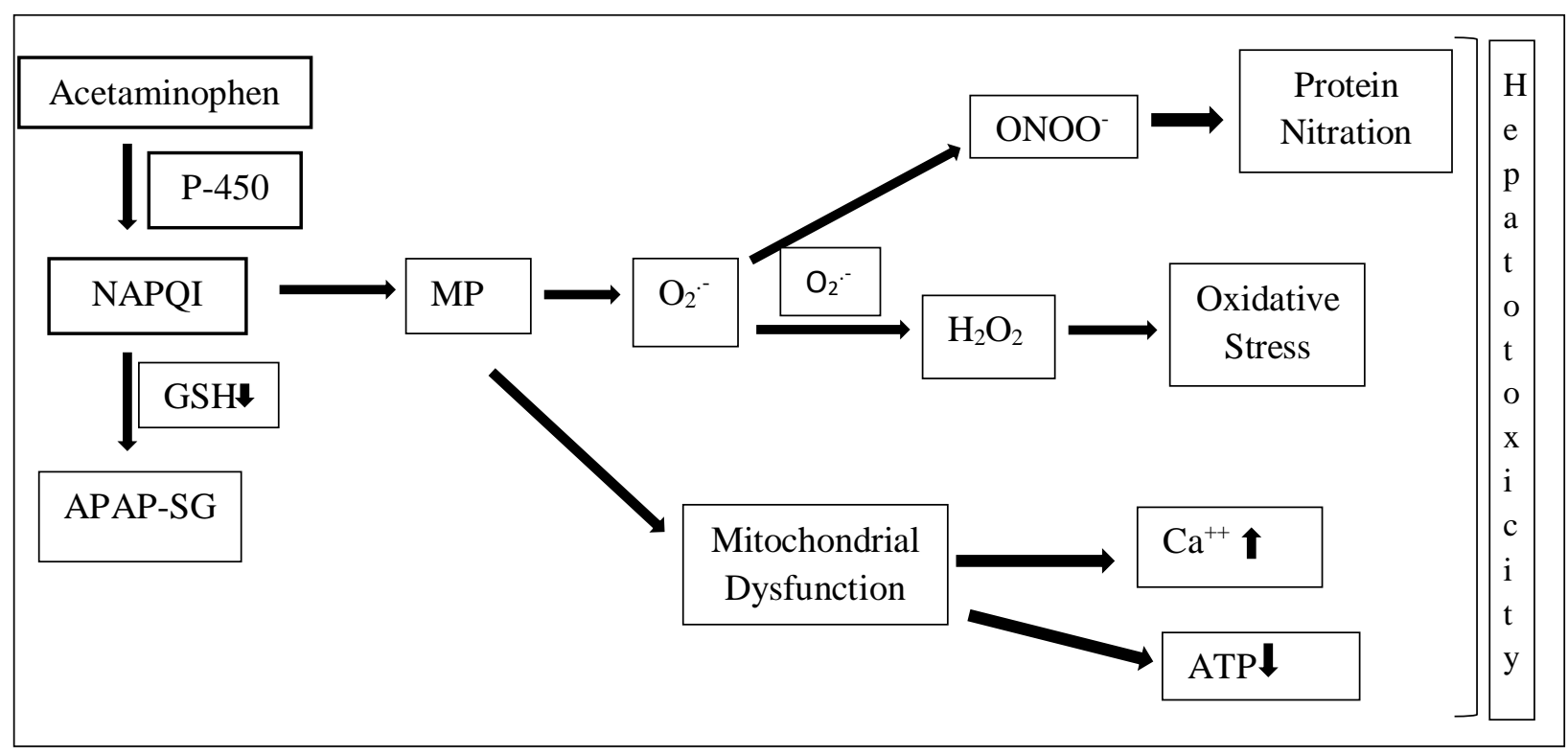

Fig. 1. Acetaminophen (APAP) metabolism and induction of hepatotoxicity

As the redox reaction is directly involved with hepatotoxicity, antioxidants application could be a potent alternative. Antioxidants are generally known as substances or compounds that have free radical scavenging capacity while inhibiting oxidative progression (Antioxidants: In Depth. NCCIH. 2010). Different naturally-derived compounds like organosulfur compounds, triterpenoids, sulphoraphae, resveratrol, saponins, lipids and different acids are more likely to act as potent antioxidants (Adeyemi et al., 2018; Atolani et al., 2019; 2020; Wang et al., 1996; Kumari \& Kakkar, 2012; Noh et al., 2015; Du et al., 2015; $\mathrm{Xu}$ et al., 2017; Pang et al., 2016; Elshazly et al., 2014). These compounds generally inhibit the acetaminophen-induced oxidative reaction in the liver and confer hepatoprotection. This review aims to enlighten the capacity of 
naturally-derived antioxidants against APAPinduced hepatotoxicity.

\section{Hepatoprotective activity of naturally- derived antioxidants:}

Several naturally derived substances showed great antioxidant activity when tested on animals with APAP-induced liver toxicity. Kumari \& Kakkar (2012) claimed in their study that, lupeol $(150 \mathrm{mg} / \mathrm{kg})$, a naturally occurring triterpenoid derived from olive, mango, crataeva, strawberry, and fig reduced oxidative damage by scavenging free radicals and prevented alteration in the antioxidant defense, inhibited depolarization of the mitochondria, prevented down-regulation of $\mathrm{Bcl}-2$, upregulation of Bax and activation of caspases, prevented DNA damage and cell death in rats (Fig. 2.). Honey is another natural substance derived from the floral nectar that prevented an increase of the serum levels of hepatic enzyme markers, reduce both oxidative stress and inflammatory cytokines thus confirming hepatoprotection (Galal et al., 2012). Different acids derived from natural sources also have great antioxidant activity when tested against APAP-induced hepatotoxicity. Arjunolic acid (AA) found in the bark of Terminalia arjunapotentially inhibited P450-mediated APAP bio-activation, and c-Jun N-terminal kinase (JNK)-mediated activation of mitochondrial permeabilization (Ghosh et al., 2010). Alpha-Lipoic acid another naturally derived compound is found to have hepatoprotective activity at a dose of 20 or 100 $\mathrm{mg} / \mathrm{kg}$ (Elshazly et al., 2014). Caffeic acid generally found in coffee decreases Kcap 1 expression, inhibits binding of Kcap 1 to nuclear factor erythroid 2-related factor 2 (Nrf2) which activates Nrf2, increases expression of anti-oxidative signals including heme oxygenase-1 (HO-1), NAD(P)H Quinone Dehydrogenase 1 (NQO1), thus protects the liver from APAP-induced toxicity (Pang et al., 2016).

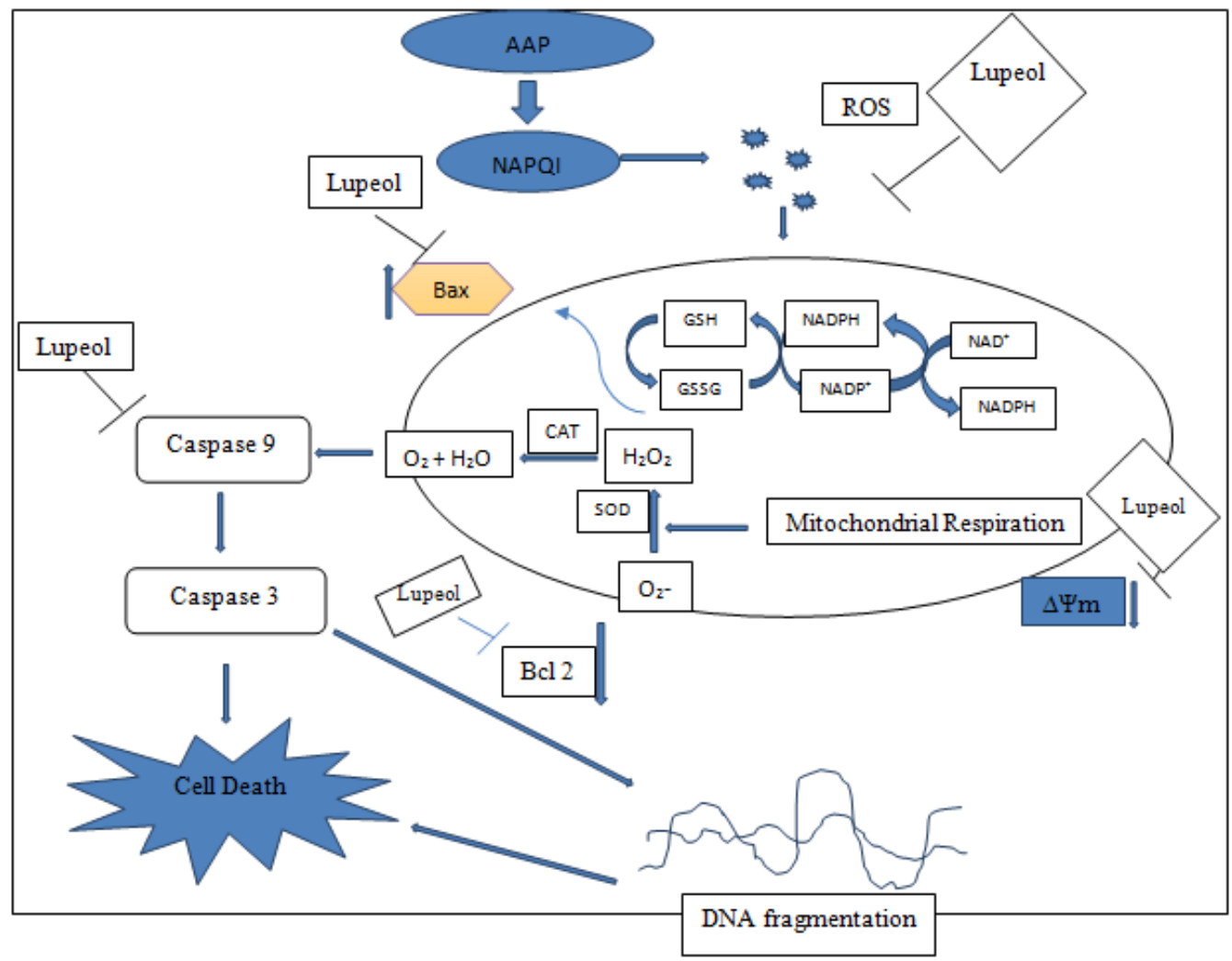

Fig. 2. Protective effect of lupeol in acetaminophen induced hepatotoxicity 
Extracts of different fruits and plants are reported to have free-radical scavenging capacity. Yen et al (2008), reported that ethanolic extract of Cuscuta chinensis (CE) and nanoparticles are potential antioxidants as they enhanced antioxidant enzymes superoxide dismutase (SOD), catalase (CAT), glutathione peroxidase (GPx), and diminished lipid peroxidation (MDA) that resulted in hepatoprotective and antioxidant activity. Polyphenol extract of Hibiscus sabdariffa L.
(HPE) at a dose of 100,200 , or $300 \mathrm{mg} / \mathrm{kg}$ reduced APAP-induced death of $\mathrm{BABL} / \mathrm{c}$ in normal liver cells (BNLs), restored lost mitochondrial potency, and improved antioxidative status (Lee et al., 2012). Polyphenol enriched fraction from Folium Microcos (FM) also acts as a great antioxidant and safeguards the liver (Wu et al., 2017). A detailed summary of the hepatoprotective tendencies of some naturally derived molecules is presented in Table 1.

Table 1. Some reported hepatoprotective potencies of naturally-derived antioxidants

\begin{tabular}{|c|c|c|c|c|}
\hline Antioxidants & Sources & $\begin{array}{l}\text { Dose/ } \\
\text { concentration } \\
(\mathbf{R} / \mathbf{A})\end{array}$ & $\begin{array}{l}\text { Protective effect } \\
\text { (possible mechanism } \\
\text { of action) }\end{array}$ & Reference \\
\hline $\begin{array}{l}\text { Fresh garlic } \\
\text { homogenates } \\
\text { (FGH) and related } \\
\text { organosulfur } \\
\text { compounds }\end{array}$ & Garlic bulbs & 2.5 or $5.0 \mathrm{~g} / \mathrm{kg}$ & $\begin{array}{l}\text { Inhibited P450 2E1- } \\
\text { mediated APAP bio- } \\
\text { activation displays } \\
\text { protective activity. }\end{array}$ & Wang et al., 1996 \\
\hline $\begin{array}{l}\text { Cuscuta chinensis } \\
\text { ethanolic extract } \\
\text { (CE) \& Cuscuta } \\
\text { chinensis } \\
\text { nanoparticles (CN) }\end{array}$ & $\begin{array}{l}\text { Seeds of Cuscuta } \\
\text { chinensis Lam. } \\
\text { (Convolvulaceae) }\end{array}$ & $\begin{array}{l}125 \& 250 \\
\mathrm{mg} / \mathrm{kg} \text { for } \mathrm{CE} \\
25 \& 50 \mathrm{mg} / \mathrm{kg} \\
\text { for } \mathrm{CN}\end{array}$ & $\begin{array}{l}\text { Enhanced antioxidant } \\
\text { enzymes (SOD, CAT, } \\
\text { GPx), and diminished } \\
\text { lipid peroxidation } \\
\text { (MDA) results in } \\
\text { hepato-protective and } \\
\text { antioxidant activity. }\end{array}$ & Yen et al., 2008 \\
\hline Sesamol & Sesame oil & $10 \mathrm{mg} / \mathrm{kg}$, i.p. & $\begin{array}{l}\text { Maintained } \\
\text { mitochondrial } \\
\text { aconitase activity in } \\
\text { the liver, ferrous ions } \\
\left(\mathrm{Fe}^{2+}\right) \text {, hydrogen } \\
\text { peroxide levels, and } \\
\text { inhibited hydroxyl- } \\
\text { radical-associated lipid } \\
\text { peroxidation and } \\
\text { hepatic injury. }\end{array}$ & $\begin{array}{l}\text { Chandrasekan et } \\
\text { al., } 2009\end{array}$ \\
\hline Spirulina fusiformis & $\begin{array}{l}\text { Spirulina } \\
\text { fusiformis }\end{array}$ & $\begin{array}{l}800 \mathrm{mg} / \mathrm{kg} / \mathrm{b} \text {. } \\
\text { wt }\end{array}$ & $\begin{array}{l}\text { Decreased liver marker } \\
\text { enzymes activity, } \\
\text { tumor necrosis factor- } \\
\text { alpha (TNF- } \alpha \text { ), and } \\
\text { lipid peroxidation level } \\
\text { with increased } \\
\text { antioxidant status. }\end{array}$ & Sabina et al., 2009 \\
\hline $\begin{array}{l}\text { Anthocyanin } \\
\text { fraction }(\mathrm{AF})\end{array}$ & $\begin{array}{l}\text { Purple fleshed } \\
\text { sweet potato }\end{array}$ & $800 \mathrm{mg} / \mathrm{kg}$ & $\begin{array}{l}\text { Blocked bio-activation } \\
\text { of APAP by inhibiting } \\
\text { CYP2E1 activity, up- } \\
\text { regulated GSH level, } \\
\text { and glutathione (GST) }\end{array}$ & Choi et al., 2009 \\
\hline
\end{tabular}




\begin{tabular}{|c|c|c|c|c|}
\hline & & & $\begin{array}{l}\text { activity which } \\
\text { increases free radical } \\
\text { scavenging capacity } \\
\text { and inhibits lipid } \\
\text { peroxidation. }\end{array}$ & \\
\hline Arjunolic acid (AA) & $\begin{array}{l}\text { Bark of } \\
\text { Terminalia arjuna }\end{array}$ & $80 \mathrm{mg} / \mathrm{kg}$ & $\begin{array}{l}\text { Inhibited P450- } \\
\text { mediated APAP bio- } \\
\text { activation and JNK- } \\
\text { mediated activation } \\
\text { inhibition of } \\
\text { mitochondrial } \\
\text { permeabilization. }\end{array}$ & Ghosh et al., 2010 \\
\hline $\begin{array}{l}\text { Curcumin (CUR; } \\
\text { diferuloylmethane) }\end{array}$ & Curcuma longa & $17 \mathrm{mg} / \mathrm{kg}$ & $\begin{array}{l}\text { Blockage of APAP } \\
\text { induced oxidative } \\
\text { stress by decreasing } \\
\text { the several pro-injury } \\
\text { parameters (alanine } \\
\text { aminotransferase } \\
\text { (ALT), nitrate/ nitrite } \\
\text { levels, lipid } \\
\text { peroxidation, DNA } \\
\text { fragmentation), and } \\
\text { several protective } \\
\text { parameters (GSH } \\
\text { content, SOD activity). }\end{array}$ & Bulku et al., 2012 \\
\hline Honey & Honey & $\begin{array}{l}5,10 \text { and } 20 \\
\mathrm{~g} / \mathrm{kg}\end{array}$ & $\begin{array}{l}\text { Prevention of increase } \\
\text { in the serum levels of } \\
\text { hepatic enzyme } \\
\text { markers, reduction in } \\
\text { both oxidative stress } \\
\text { and inflammatory } \\
\text { cytokines. }\end{array}$ & Galal et al., 2012 \\
\hline $\begin{array}{l}\text { Polyphenol extract } \\
\text { of Hibiscus } \\
\text { sabdariffa L. (HPE) }\end{array}$ & $\begin{array}{l}\text { Hibiscus } \\
\text { sabdariffa L. }\end{array}$ & $\begin{array}{l}100,200 \text { or } \\
300 \mathrm{mg} / \mathrm{kg}\end{array}$ & $\begin{array}{l}\text { Reduced APAP- } \\
\text { induced death of } \\
\text { BABL/c in normal } \\
\text { liver cells (BNLs), } \\
\text { restored lost } \\
\text { mitochondrial potency, } \\
\text { and improved anti- } \\
\text { oxidative status. }\end{array}$ & Lee et al., 2012 \\
\hline $\begin{array}{l}\text { Lupeol, a naturally } \\
\text { occurring } \\
\text { triterpenoid }\end{array}$ & $\begin{array}{l}\text { Olive, mango, } \\
\text { crataeva, } \\
\text { strawberry, and } \\
\text { fig. }\end{array}$ & $150 \mathrm{mg} / \mathrm{kg}$ & $\begin{array}{l}\text { Reduced oxidative } \\
\text { damage by scavenging } \\
\text { free radicals and } \\
\text { preventing alteration in } \\
\text { the antioxidant } \\
\text { defense, inhibited } \\
\text { depolarization of the } \\
\text { mitochondria, } \\
\text { prevented down- } \\
\text { regulation of Bcl-2, up- } \\
\text { regulation of Bax and } \\
\text { activation of caspases, } \\
\text { prevented DNA } \\
\text { damage and cell death. }\end{array}$ & $\begin{array}{l}\text { Kumari \& Kakkar, } \\
2012\end{array}$ \\
\hline
\end{tabular}




\begin{tabular}{|c|c|c|c|c|}
\hline Red ginseng extract & $\begin{array}{l}\text { Roots of Panax } \\
\text { ginseng C. A } \\
\text { Meyer }\end{array}$ & $\begin{array}{l}10,30,100, \\
300,500 \mathrm{mg} / \mathrm{kg}\end{array}$ & $\begin{array}{l}\text { Suppressed } \\
\text { hepatotoxicity, } \\
\text { suppressed hepatic } \\
\text { CYP2E1 leading to } \\
\text { high retention of intact } \\
\text { APAP in plasma and } \\
\text { GSTA2 gene induction } \\
\text { with transcriptional } \\
\text { activation of Nrf2 and/ } \\
\text { or C/ EBPb } \\
\text { downstream of } \\
\text { multiple signaling } \\
\text { pathways that facilitate } \\
\text { conjugation of GSH } \\
\text { with NAPQI. }\end{array}$ & Gum \& Cho, 2013 \\
\hline Silymarin & $\begin{array}{l}\text { Silybum } \\
\text { marianum (milk } \\
\text { thistle) }\end{array}$ & $100 \mathrm{mg} / \mathrm{kg}$ & $\begin{array}{l}\text { Inhibition of migrating } \\
\text { neutrophils, protection } \\
\text { against GSH depletion } \\
\text { that elevates nitric } \\
\text { oxide (NO) levels in } \\
\text { tissue along with } \\
\text { antioxidant and free } \\
\text { radical scavenging } \\
\text { properties. }\end{array}$ & Bektur et al., 2013 \\
\hline Ginger & $\begin{array}{l}\text { Zingiber officinale } \\
\text { Roscoe }\end{array}$ & $100 \mathrm{mg} / \mathrm{kg}$ & $\begin{array}{l}\text { Reduced hepatic } \\
\text { marker enzymes } \\
\text { (aspartate } \\
\text { aminotransferase, } \\
\text { serum alanine } \\
\text { aminotransferase, and } \\
\text { arginase) and total } \\
\text { bilirubin in plasma, } \\
\text { improved paracetamol } \\
\text { (PARA)-induced } \\
\text { oxidative stress by } \\
\text { inhibiting lipid } \\
\text { peroxidation } \\
\text { malondialdehyde } \\
\text { (MDA), restored } \\
\text { triacylglycerols } \\
\text { (TAGs), and total } \\
\text { protein levels. }\end{array}$ & $\begin{array}{l}\text { Abdel-Azeem et } \\
\text { al., } 2013\end{array}$ \\
\hline Apigenin & $\begin{array}{l}\text { Parsley, onions, } \\
\text { oranges, tea, } \\
\text { and chamomile }\end{array}$ & $\begin{array}{l}100 \text { and } 200 \\
\mathrm{mg} / \mathrm{kg}\end{array}$ & $\begin{array}{l}\text { Increased hepatic } \\
\text { glutathione reductase } \\
\text { (GR) activity, reduced } \\
\text { GSH content, and } \\
\text { decreased hepatic } \\
\text { malondialdehyde } \\
\text { content. }\end{array}$ & Yang et al., 2013 \\
\hline $\begin{array}{l}\text { Glossogyne } \\
\text { tenuifolia (GT) } \\
\text { Cassini }\end{array}$ & $\begin{array}{l}\text { Glossogyne } \\
\text { tenuifolia }\end{array}$ & $\begin{array}{l}100 \text { or } 300 \\
\mathrm{mg} / \mathrm{kg}\end{array}$ & $\begin{array}{l}\text { Decreased ALT, } \\
\text { aspartate } \\
\text { aminotransferase } \\
(\text { AST) in serum, } \\
\text { alleviated GSH } \\
\text { depletion, and }\end{array}$ & Tien et al., 2014 \\
\hline
\end{tabular}




\begin{tabular}{|c|c|c|c|c|}
\hline & & & $\begin{array}{l}\text { inhibited lipid } \\
\text { peroxidation leads to } \\
\text { free radical scavenging } \\
\text { and antioxidant } \\
\text { activity. }\end{array}$ & \\
\hline Alpha-Lipoic acid & $\begin{array}{l}\text { Naturally found in } \\
\text { plants and } \\
\text { animals. }\end{array}$ & $\begin{array}{l}20 \text { or } 100 \\
\mathrm{mg} / \mathrm{kg}\end{array}$ & $\begin{array}{l}\text { Reduction in APAP- } \\
\text { induced liver injury is } \\
\text { seen by restoring the } \\
\text { changes in ALT, total } \\
\text { protein, GSH, MDA, } \\
\text { GSH synthase, } \\
\text { crystathionine } \beta \text { - } \\
\text { synthase, NADPH } \\
\text { oxidae, and nuclear } \\
\text { factor kappa B (NF- } \\
\kappa B) \text { towards control } \\
\text { value. Also improves } \\
\text { the hepatic } \\
\text { histopathology with an } \\
\text { increase in the } \\
\text { expression of HO-1, } \\
\text { Nrf2. }\end{array}$ & $\begin{array}{l}\text { Elshazly et al., } \\
2014\end{array}$ \\
\hline Aloe vera & Aloe vera & $150 \mathrm{mg} / \mathrm{kg}$ & $\begin{array}{l}\text { Improved level of } \\
\text { serum alanine } \\
\text { aminotransferase, } \\
\text { hepatic MDA, number } \\
\text { of interleukin-12 (IL- } \\
\text { 12), and interleukin-18 } \\
\text { (IL-18) positive- } \\
\text { stained cells, and } \\
\text { hepatic GSH along } \\
\text { with improved liver } \\
\text { histopathology. }\end{array}$ & $\begin{array}{l}\text { Werawatgann et } \\
\text { al., } 2014\end{array}$ \\
\hline $\begin{array}{l}\text { Sulphoraphae } \\
\text { (SFN) }\end{array}$ & $\begin{array}{l}\text { Cruciferous } \\
\text { vegetables of the } \\
\text { genus Brassica } \\
\text { such as } \\
\text { cauliflower, kale, } \\
\text { broccoli, cole } \\
\text { crops, cabbage, } \\
\text { collards, brussels } \\
\text { sprout, mustard, } \\
\text { cress, and even } \\
\text { radish. }\end{array}$ & $5 \mathrm{mg} / \mathrm{kg}$ & $\begin{array}{l}\text { Antioxidant activity } \\
\text { against APAP-induced } \\
\text { liver injuries by } \\
\text { blocking ROS } \\
\text { generation, GSH } \\
\text { depletion, and lipid } \\
\text { peroxidation followed } \\
\text { by up-regulation of } \\
\text { Nrf2-targeted } \\
\text { cytoprotective genes } \\
\text { such as HO-1. }\end{array}$ & Noh et al., 2015 \\
\hline Resveratrol & Skin of grape & $50 \mathrm{mg} / \mathrm{kg}$ & $\begin{array}{l}\text { Reduced } \\
\text { hepatotoxicity by } \\
\text { scavenging } \\
\text { peroxynitrite and } \\
\text { preventing apoptosis- } \\
\text { inducing factor (AIF), } \\
\text { EndoG release from } \\
\text { mitochondria, and } \\
\text { subsequent nuclear } \\
\text { DNA fragmentation. }\end{array}$ & Du et al., 2015 \\
\hline
\end{tabular}


RAHAMAN et al.

\begin{tabular}{|c|c|c|c|c|}
\hline Caffeic acid & $\begin{array}{l}\text { Coffee, some } \\
\text { fruits, and } \\
\text { traditional } \\
\text { Chinese } \\
\text { medicines }\end{array}$ & $10,30 \mathrm{mg} / \mathrm{kg}$ & $\begin{array}{l}\text { Decreased Kcap } 1 \\
\text { expression, inhibited } \\
\text { binding of Kcap } 1 \text { to } \\
\text { Nrf2 which activates } \\
\text { Nrf2, increased } \\
\text { expression of anti- } \\
\text { oxidative signals } \\
\text { including HO-1, } \\
\text { NQO1. }\end{array}$ & Pang et al., 2016 \\
\hline Black ginseng (BG) & $\begin{array}{l}\text { Roots of Panax } \\
\text { ginseng C. A } \\
\text { Meyer }\end{array}$ & $600 \mathrm{mg} / \mathrm{kg}$ & $\begin{array}{l}\text { Decreased level of } \\
\text { ALT, AST, decreased } \\
\text { lipid peroxidation, } \\
\text { increased hepatic } \\
\text { antioxidants GSH, } \\
\text { apoptotic pathway } \\
\text { suppression by } \\
\text { increasing Bcl-2, and } \\
\text { decreasing Bax protein } \\
\text { expression which } \\
\text { resulted in inhibited } \\
\text { APAP-induced } \\
\text { necrosis and } \\
\text { inflammatory } \\
\text { infiltration in the liver } \\
\text { tissue. }\end{array}$ & Hu et al., 2017 \\
\hline $\begin{array}{l}\text { Saponins } \\
\text { (ginsenosides) }\end{array}$ & $\begin{array}{l}\text { Leaves of Panax } \\
\text { quinquefolius } \\
\text { (PQS) }\end{array}$ & $\begin{array}{l}150 \text { and } 300 \\
\mathrm{mg} / \mathrm{kg}\end{array}$ & $\begin{array}{l}\text { Ameliorated oxidative } \\
\text { stress via lipid } \\
\text { peroxidation } \\
\text { suppression, down- } \\
\text { regulation of pro- } \\
\text { inflammatory factors } \\
\text { disrupted apoptotic } \\
\text { signal pathway by Bcl- } \\
2 \text { overexpression, and } \\
\text { Bax low-expression, } \\
\text { prevented caspase-3 } \\
\text { release. }\end{array}$ & Xu et al., 2017 \\
\hline $\begin{array}{l}\text { Polyphenol } \\
\text { enriched fraction } \\
\text { from Folium } \\
\text { Microcos (FM) }\end{array}$ & $\begin{array}{l}\text { Leaves of } \\
\text { Microcos } \\
\text { paniculata L. }\end{array}$ & $\begin{array}{l}100,200 \& 400 \\
\mathrm{mg} / \mathrm{kg} \text { body } \\
\text { weight }\end{array}$ & $\begin{array}{l}\text { Modified ROS/ } \\
\text { mitogen-activated } \\
\text { protein kinase } \\
\text { (MAPK)/ apoptosis } \\
\text { axis, and Nrf2- } \\
\text { mediated antioxidant } \\
\text { response by four } \\
\text { phenolic compounds: } \\
\text { narcissin, } \\
\text { isorhamnetin-3-O- } \beta \text {-D- } \\
\text { glucoside, isovitexin, } \\
\text { and vitexin. }\end{array}$ & Wu et al., 2017 \\
\hline Nutmeg & $\begin{array}{l}\text { Kernel extract of } \\
\text { Myristica } \\
\text { fragrans }\end{array}$ & $300 \mathrm{mg} / \mathrm{kg}$ & $\begin{array}{l}\text { Suppressed oxidative } \\
\text { stress, inflammation, } \\
\text { and apoptosis, } \\
\text { promoted } \\
\text { Nrf2/antioxidant } \\
\text { responsive element }\end{array}$ & Dkhil et al., 2019 \\
\hline
\end{tabular}




\begin{tabular}{|c|c|c|c|c|}
\hline & & & $\begin{array}{l}\text { (ARE) pathway which } \\
\text { leads to } \\
\text { hepatoprotection. }\end{array}$ & \\
\hline Sonneratia apetala & $\begin{array}{l}\text { Sonneratia } \\
\text { apetala }\end{array}$ & $\begin{array}{l}100,200 \& 400 \\
\mathrm{mg} / \mathrm{kg}\end{array}$ & $\begin{array}{l}\text { Decreased ALT, AST } \\
\text { level in serum, reduced } \\
\text { MDA in the liver, } \\
\text { increased glutathione } \\
\text { (GSH), glutathione } \\
\text { peroxidase (GPx) } \\
\text { activity, enhanced } \\
\text { catalase and } \\
\text { antioxidant capacity, } \\
\text { and inhibited TNF- } \alpha \text {, } \\
\text { IL-6, myeloperoxidase } \\
\text { (MPO) formation in } \\
\text { liver inhibits APAP } \\
\text { induced liver injury. }\end{array}$ & Liu et al., 2019 \\
\hline
\end{tabular}

\section{Conclusions}

The liver is a major organ of our body that performs several functions including metabolism and detoxification of substances. Acetaminophen is a globally used drug for different health issues. Exceeding the therapeutic level, a higher dose may often induce severe toxicity in the liver. Researchers from time to time have discovered the direct connection of redox reaction with hepatotoxicity. However, this situation can be controlled by using antioxidants that scavenge free radicals produced during the oxidative reaction. As enumerated in this review, antioxidants derived from natural sources like saponins, acids, triterpenoids, polyphenols seem to have a significant effect against APAPinduced hepatotoxicity.

\section{Conflict of Interest}

The authors declare that the research was conducted in the absence of any commercial or financial relationships that could be construed as a potential conflict of interest.

\section{Acknowledgments}

No funding was received for the work.

\section{References}

1. Abdel-Azeem AS, Hegazy AM, Ibrahim KS, Farrag ARH, El-Sayed EM (2013) Hepatoprotective, antioxidant, and ameliorative effects of ginger (Zingiber officinale Roscoe) and vitamin $\mathrm{E}$ in acetaminophen treated rats. Journal of dietary supplements 10(3):195-209.

2. Almeer RS, Alarifi S, Alkahtani S, Ibrahim SR, Ali D, Moneim A (2018) The potential hepatoprotective effect of royal jelly against cadmium chloride-induced hepatotoxicity in mice is mediated by suppression of oxidative stress and upregulation of Nrf2 expression. Biomedicine \& Pharmacotherapy 106:1490-1498.

3. Adeyemi OS, Atolani O, Banerjee P, Arolasafe G, Preissner R, Etukudoh P, Ibraheem O (2018) Computational and experimental validation of antioxidant properties of synthesized bioactive ferulic acid derivatives. International Journal of Food Properties 21(1):86-88.

4. Antioxidants: In Depth. NCCIH (2010) Archived from the original on 25 August 2018. Retrieved 20 June 2018. 
5. Atolani O, Areh ET, Oguntoye OS, Zubair MF, Fabiyi OA, Oyegoke RA, Tarigha DE, Adamu N, Adeyemi OS, Kambizi L, Olatunji GA (2020) Chemical characterization, antioxidant, cytotoxicity, anti-toxoplasma gondii and antimicrobial potentials of the Citrus sinensis seed oil for sustainable cosmeceutical production. Heliyon, 6(2):e03399.

6. Atolani O, Oguntoye H, Areh ET, Adeyemi OS, Kambizi L (2019) Chemical composition, anti-toxoplasma, cytotoxicity, antioxidant, and anti-inflammatory potential of Cola gigantea seed oil. Pharmaceutical Biology 57(1):154-160.

7. Bektur NE, Sahin E, Baycu C, Unver G (2016) Protective effects of silymarin against acetaminophen-induced hepatotoxicity and nephrotoxicity in mice. Toxicology and Industrial Health 32(4):589-600.

8. Bessems JG, Vermeulen NP (2001) Paracetamol (acetaminophen)-induced toxicity: molecular and biochemical mechanisms, analogues and protective approaches. Critical reviews in toxicology 31(1):55-138.

9. Bulku EJ, Stohs S, Cicero L, Brooks T, Halley H, Ray DS (2012) Curcumin exposure modulates multiple pro-apoptotic and anti-apoptotic signaling pathways to antagonize acetaminophen-induced toxicity. Current neurovascular research 9(1):58-71.

10. Chandrasekaran VRM, Hsu DZ, Liu MY (2009) The protective effect of sesamol against mitochondrial oxidative stress and hepatic injury in acetaminophen-overdosed rats. Shock 32(1):89-93.

11. Choi JH, Choi CY, Lee KJ, Hwang YP, Chung YC, Jeong HG (2009) Hepatoprotective effects of an anthocyanin fraction from purple-fleshed sweet potato against acetaminophen-induced liver damage in mice. Journal of medicinal food 12(2):320-326.

12. Corcoran GB, Racz WJ, Smith CV, Mitchell JR (1985) Effects of Nacetylcysteine on acetaminophen covalent binding and hepatic necrosis in mice. Journal of Pharmacology and Experimental Therapeutics 232(3):864-872.

13. Dargan P, Jones A (2002) Paracetamol: balancing risk against benefit. Qjm 95(12):831-832.

14. Dkhil MA, Abdel Moneim AE, Hafez TA, Mubaraki MA, Mohamed WF, Thagfan FA, Al-Quraishy S (2019) Myristica fragrans kernels prevent paracetamolinduced hepatotoxicity by inducing antiapoptotic genes and Nrf2/HO-1 pathway. International journal of molecular sciences 20(4):993.

15. Du K, McGill MR, Xie Y, Bajt ML, Jaeschke H (2015) Resveratrol prevents protein nitration and release of endonucleases from mitochondria during acetaminophen hepatotoxicity. Food and Chemical Toxicology 81:62-70.

16. Elshazly SM, El-Moselhy MA, Barakat W (2014) Insights in the mechanism underlying the protective effect of $\alpha$-lipoic acid against acetaminophen-hepatotoxicity. European journal of pharmacology, 726:116-123.

17. Esterline RL, Ray SD, Ji S (1989) Reversible and irreversible inhibition of hepatic mitochondrial respiration by acetaminophen and its toxic metabolite, $\mathrm{N}$ acetyl-p-benzoquinoneimine (NAPQI). Biochemical pharmacology 38(14):23872390.

18. Galal RM, Zaki HF, Seif ENMM, Agha AM (2012) Potential protective effect of honey against paracetamol-induced hepatotoxicity. Archives of Iranian medicine 15(11):674-80. 
19. Ghosh J, Das J, Manna P, Sil PC (2010) Arjunolic acid, a triterpenoid saponin, prevents acetaminophen (APAP)-induced liver and hepatocyte injury via the inhibition of APAP bioactivation and JNKmediated mitochondrial protection. Free Radical Biology and Medicine 48(4):535553.

20. Gum SI, Cho MK (2013) Korean red ginseng extract prevents APAP-induced hepatotoxicity through metabolic enzyme regulation: The role of ginsenoside $\operatorname{Rg} 3$, a protopanaxadiol. Liver International 33(7):1071-1084.

21. Hazai E, Vereczkey L, Monostory K (2002) Reduction of toxic metabolite formation of acetaminophen. Biochemical and Biophysical Research Communications 291(4):1089-1094.

22. Hinson JA, Bucci TJ, Irwin LK, Michael SL, Mayeux PR (2002) Effect of inhibitors of nitric oxide synthase on acetaminopheninduced hepatotoxicity in mice. Nitric Oxide 6(2):160-167.

23. Hinson JA, Reid AB, McCullough SS, James LP (2004) Acetaminophen-induced hepatotoxicity: role of metabolic activation, reactive oxygen/nitrogen species, and mitochondrial permeability transition. Drug metabolism reviews 36(3-4):805-822.

24. Hinson JA, Roberts DW, James LP (2010) Mechanisms of acetaminophen-induced liver necrosis. In: Adverse drug reactions, Springer, Berlin, Heidelberg. pp 369-405.

25. Hu JN, Liu Z, Wang Z, Li XD, Zhang LX, Li W, Wang YP (2017) Ameliorative effects and possible molecular mechanism of action of black ginseng (Panax ginseng) on acetaminophen-mediated liver injury. Molecules 22(4):664.

26. Jaeschke H, Williams CD, Ramachandran A, Bajt ML (2012) Acetaminophen hepatotoxicity and repair: the role of sterile inflammation and innate immunity. Liver International 32(1):8-20.

27. James LP, Mayeux PR, Hinson JA (2003) Acetaminophen-induced hepatotoxicity. Drug metabolism and disposition 31(12):1499-1506.

28. Kon K, Ikejima K, Okumura K, Aoyama T, Arai K, Takei Y, Sato N (2007) Role of apoptosis in acetaminophen hepatotoxicity. Journal of gastroenterology and hepatology 22:S49-S52. doi: 10.1111/j.14401746.2007.04962.x

29. Kumari A，Kakkar P (2012) Lupeol prevents acetaminophen-induced in vivo hepatotoxicity by altering the $\mathrm{Bax} / \mathrm{Bcl}-2$ and oxidative stress-mediated mitochondrial signaling cascade. Life sciences 90(15-16):561-570.

30. Larson AM (2007) Acetaminophen hepatotoxicity. Clinics in liver disease 11(3):525-548.

31. Lee CH, Kuo CY, Wang CJ, Wang CP, Lee YR, Hung CN, Lee HJ (2012) A polyphenol extract of Hibiscus sabdariffa L. ameliorates acetaminophen-induced hepatic steatosis by attenuating the mitochondrial dysfunction in vivo and in vitro. Bioscience, biotechnology, and biochemistry 76(4):646-651.

32. Liu J, Luo D, Wu Y, Gao C, Lin G, Chen J, Su Z (2019) The protective effect of Sonneratia apetala fruit extract on acetaminophen-induced liver injury in mice. Evidence-Based Complementary and Alternative Medicine. https://doi.org/10.1155/2019/6919834

33. McGill MR, Sharpe MR, Williams CD, Taha M, Curry SC, Jaeschke H (2012) The mechanism underlying acetaminopheninduced hepatotoxicity in humans and mice involves mitochondrial damage and nuclear DNA fragmentation. The Journal of clinical investigation 122(4):1574-1583. 
34. Michael SL, Pumford NR, Mayeux PR, Niesman MR, Hinson JA (1999) Pretreatment of mice with macrophage inactivators decreases acetaminophen hepatotoxicity and the formation of reactive oxygen and nitrogen species. Hepatology 30(1):186-195.

35. Nelson SD (1990) Molecular mechanisms of the hepatotoxicity caused by acetaminophen. In: Seminars in liver disease, Thieme Medical Publishers, Inc., Vol. 10, No. 04, pp 267-278.

36. Noh JR, Kim YH, Hwang JH, Choi DH, Kim KS, Oh WK, Lee CH (2015) Sulforaphane protects against acetaminophen-induced hepatotoxicity. Food and Chemical Toxicology 80:193200.

37. Pang C, Zheng Z, Shi L, Sheng Y, Wei H, Wang Z, Ji L (2016) Caffeic acid prevents acetaminophen-induced liver injury by activating the Keap1-Nrf2 antioxidative defense system. Free Radical Biology and Medicine 91:236-246.

38. Ray SD, Corcoran GB (2009) Apoptosis and cell death. General and Applied Toxicology 247-312.

39. Sabina E, Samuel J, RajappaRamya S, Patel S, Mandal N, Pranatharthiiharan P, Mishra PP, Rasool M (2009) Hepatoprotective and antioxidant potential of Spirulina fusiformis on acetaminophen-induced hepatotoxicity in mice. IJIB 6(1):1-5.

40. Tien YH, Chen BH, Wang Hsu GS, Lin WT, Huang JH, Lu YF (2014) Hepatoprotective and anti-oxidant activities of Glossogyne tenuifolia against acetaminophen-induced hepatotoxicity in mice. The American journal of Chinese medicine 42(06): 1385-1398.

41. Wang EJ, Li Y, Lin M, Chen L, Stein AP, Reuhl KR, Yang CS (1996) Protective effects of garlic and related organosulfur compounds on acetaminophen-induced hepatotoxicity in mice. Toxicology and applied pharmacology 136(1):146-154.

42. Werawatganon D, Linlawan S, Thanapirom K, Somanawat K, Klaikeaw N, Rerknimitr R, Siriviriyakul P (2014) Aloe vera attenuated liver injury in mice with acetaminophen-induced hepatitis. BMC complementary and alternative medicine 14(1):229.

43. Wu H, Zhang G, Huang L, Pang H, Zhang N, Chen Y, Wang G (2017) Hepatoprotective effect of polyphenolenriched fraction from Folium Microcos on oxidative stress and apoptosis in acetaminophen-induced liver injury in mice. Oxid Med Cell Longev 2017:3631565. doi: 10.1155/2017/3631565.

44. Xu XY, Hu JN, Liu Z, Zhang R, He YF, Hou W, Wang ZQ, Yang G, Li W (2017) Saponins (Ginsenosides) from the leaves of Panax quinquefolius ameliorated acetaminophen-induced hepatotoxicity in mice. Journal of agricultural and food chemistry 65(18):3684-3692.

45. Yang J, Wang XY, Xue J, Gu ZL, Xie ML (2013) Protective effect of apigenin on mouse acute liver injury induced by acetaminophen is associated with increment of hepatic glutathione reductase activity. Food \& function, 4(6):939-943.

46. Yen FL, Wu TH, Lin LT, Cham TM, Lin CC (2008) Nanoparticles formulation of Cuscuta chinensis prevents acetaminopheninduced hepatotoxicity in rats. Food and chemical toxicology 46(5):1771-1777.

47. ***https://medlineplus.gov/druginfo/meds/ a681004.html\#: :text=Acetaminophen\%20i s\%20used\%20to\%20relieve,)\%2C\%20and $\% 20$ to\%20reduce\%20fever. 